\title{
Menopausal Status and Metabolic Syndrome in Women in Climacteric Period Treated at a Clinic in Southern Brazil
}

\author{
Karina Giane Mendes ${ }^{1,2}$, Heloísa Theodoro ${ }^{3}$, Alice D. Rodrigues ${ }^{3}$, Fernanda Busnello ${ }^{4}$, \\ Dino Roberto S. de Lorenzi ${ }^{2}$, Maria Teresa A. Olinto ${ }^{3,4}$ \\ ${ }^{1}$ Post-Graduate Program in Medical Sciences: Endocrinology, Federal University of Rio Grande do Sul, Porto Alegre, Brazil \\ ${ }^{2}$ University of Caxias do Sul, Caxias do Sul, Brazil \\ ${ }^{3}$ Post-Graduate Program in Collective Health, University of Vale do Rio dos Sinos, São Leopoldo, Brazil \\ ${ }^{4}$ Nutrition Department, Federal University of Health Sciences of Porto Alegre, Porto Alegre, Brazil \\ Email: mtolinto@gmail.com
}

Received November 11, 2012; revised December 13, 2012; accepted January 15, 2013

\begin{abstract}
Background: This study aims to understand the relationship between menopausal status and the presence of Metabolic Syndrome in women from 40 to 65 years, as well as to describe the distribution of each component of Metabolic Syndrome according to sample characteristics. Methods: A cross-sectional study was conducted with 551 women treated at a clinic in southern Brazil. MetS and its components were defined according to NCEP-ATP III and menopausal status as pre, peri, and post-menopause. Prevalences of menopausal status and of MetS and its components were calculated. Estimates of prevalence ratios crude and adjusted with confidence intervals of $95 \%$ were calculated by Poisson Regression with robust variance. Demographic, socioeconomic, behavioral, and reproductive characteristics were considered as potential confounding factors in multivariable models based on a conceptual framework of MetS determination. Results: The prevalence of Metabolic Syndrome in the sample was $56.1 \%\left(\mathrm{CI}_{95 \%}: 51.9\right.$ to 60.2$)$, being more common among older women (56 to 65 years), with low education, menarche $\leq 11$ years old, with three or more pregnancies and in the post-menopausal period. In multivariate analysis, there was an increase of prevalence ratios when comparing perimenopause and post-menopause with pre-menopause; however, the confidence intervals include the unit. Regarding the analysis of isolated components in the sample, the most prevalent altered components were: hypertension $(84.8 \%$; $\mathrm{CI}_{95 \%}: 81.7$ to 87.8 ), waist circumference (66.4\%; $\mathrm{CI}_{95 \%}: 62.5$ to 70.4$)$ and HDL cholesterol $\left(51.7 \% ; \mathrm{CI}_{95 \%}: 47.5\right.$ to 55.9$)$. There was a linear increase on mean blood glucose through menopausal status. Conclusions: Our study indicates variation on the distribution of MetS and each component according to menopausal status and other women characteristics. Future studies on MetS should also have foresight to use this type of approach to improve understanding and targeting of actions and programs focusing on women in this period of life.
\end{abstract}

Keywords: Metabolic Syndrome; Climacteric; Menopause; Pre-Menopause; Perimenopause; Post-Menopause

\section{Introduction}

Climacteric is defined as the period of transition from the reproductive stage to the non-reproductive stage. This period is characterized by endocrine changes, such as the hypoestrogenism, due to the decline of ovarian activity, biological changes because of decreased fertility, and clinical changes resulting from changes in the menstrual cycle and a number of factors [1]. The climacteric is divided into three periods, which define women's menopausal status. The menopausal status is established according to the menstrual cycle characteristics or the time of amenorrhea, and can be divided into pre-menopause, perimenopause and post-menopause, based on the last menstrual period (menopause) [2]. MetS is a complex disorder, characterized by a grouping of cardiovascular risk factors related to the resistance to insulin action, central obesity, dyslipidemia (increased triglycerides and decreased HDL cholesterol) and altered blood pressure [3].

It is estimated that 47 million American adults have MetS [4]. Data from Framingham's study indicates that $20 \%$ to $30 \%$ of middle-aged adults are affected by MetS [5]. In Brazil, some studies show even higher prevalence of MetS. In a 2007 study, the prevalence of MetS was $35.5 \%$ [6]. In another study, the prevalence of MetS was $29.8 \%$ and an increase in prevalence as the age increases was noticed [7]. In a study by Figueiredo Neto [8], women between 40 and 45 years had a prevalence of MetS of $14.1 \%$, while for women between 56 and 64 years the prevalence was $66.7 \%$. In this study, the increase in the prevalence of MetS in post-menopausal 
women was caused mainly by the increase of age. On the other hand, some studies have shown a relationship between menopausal status and presence of Metabolic Syndrome (MetS) regardless of age, suggesting that menopause contributes to the increase in this prevalence [9-12].

The increase in prevalence of obesity and MetS in post-menopausal women has attracted considerable interest, since women in this situation tend to develop cardiovascular diseases [13-14]. However, there are few studies exploring the relationship between menopausal status and MetS [8,9,11,12,15-20].

Thus, this study aims to understand the relationship between menopausal status and the presence of MetS in women from 40 to 65 years, as well as to describe the distribution of each component of MetS according to menopausal status and other women characteristics.

\section{Methods}

A cross-sectional study was conducted with women from 40 to 65 years treated at the clinic of Climacteric and of Gynecological Surgery, belonging to the Central Clinic of the University of Caxias do Sul (AMCE-UCS), during the period from January 2010 to May 2011. Both clinics are part of the Unified Health System and serve the population of the municipality and region. Hysterectomized women and women who were submitted to hormone replacement therapy were excluded from the study.

The sample size was calculated for the association between menopausal status and MetS. Thus, a sample of 687 women would allow identifying an odds ratio of 1.6 [9], with a confidence level of $95 \%$, a statistical power of $80 \%$ and a ratio of unexposed:exposed maintained at $2: 1$. The unexposed are the pre-menopausal women, and the exposed are the post-menopausal women.

All interviews were conducted in the clinics, prior medical consultation, by trained interviewers. A standardized, pre-coded and pre-tested questionnaire was applied, containing socioeconomic, demographic, previous reproductive and behavioral characteristic questions. The demographic characteristics evaluated were: age (40 to 45 years; 46 to 50 years; 51 to 55 years; 56 to 65 years); skin color (white or not white); and marital status (married/in union and not married). The socioeconomic characteristics evaluated were: schooling ( 0 to 4,5 to 8,9 to 11 and $\geq 12$ full years of study), family income ( 0 to 2 , 2.01 to $3.00,3.01$ to 5.00 ; $\geq 5.01$ minimum wages; value at the time: R\$ 510.00); and paid work (yes/no). Schooling was recategorized for bivariate and multivariate analysis ( 0 to $4 ; 5$ to $8 ; \geq 9$ full years of study). This recategorization occurred because of the small number of women in the category $\geq 12$ full years of study. As be- havioral characteristics, smoking and physical activity were evaluated. Smoking habit was classified as smoker, former smoker and nonsmoker. As to physical activity, the women were classified as sedentary (women who reported no physical activity) and non-sedentary (those practicing any physical activity at least once a week for at least 30 minutes) [21]. The women's previous reproductive characteristics were evaluated using the following variables: menarche $(\geq 14 ; 12-13 ; \leq 11$ years of age); number of pregnancies ( 0 to $1 ; 2$; and $\geq 3$ pregnancies).

The MetS was evaluated through each of its components: waist circumference, blood pressure, blood glucose, levels of HDL cholesterol and triglycerides. Waist circumference (WC) was measured by the survey coordinators. To this measure, a standardization training was performed in order to reduce intra- and inter-observer error. The WC was measured with a non-flexible tape measure directly on the skin at the midpoint between the last rib and the iliac crest, and the readings were made at the end of a normal expiration [22]. Abdominal obesity was obtained by the average between two WC measurements. The blood pressure levels were measured at the wrist with a validated automatic digital device (BP3AF13 ), according to the guidelines of Association for the Advancement of Medical Instrumentation (AAMI) and British Hypertension Society (BHS) [23]. The woman remained sitting, without speaking, and at rest for at least 5 minutes. This procedure was performed two non-consecutive times in a minimum interval of 5 minutes, and the average value between the two measurements was used for analysis.

Laboratory tests were collected from participants' medical records, with a limit of four months. Since the request of these tests was part of the clinic routine, most women already had them. For those women who had old tests, new tests were requested by the physician responsible for the clinic.

MetS was defined according to diagnostic criteria of NCEP-ATP III [3]. Thus, MetS would be present in women with at least three of the following criteria: waist circumference $\geq 88 \mathrm{~cm}$, blood pressure $\geq 130 \mathrm{mmHg}$ or $\geq$ $85 \mathrm{mmHg}$, fasting blood glucose $\geq 100 \mathrm{mg} / \mathrm{dl}$, triglycerides $\geq 150 \mathrm{mg} / \mathrm{dl}$, HDL cholesterol $\leq 50 \mathrm{mg} / \mathrm{dl}$ or in use of medication to treat these conditions.

For classification of menopausal status, the study participants were asked if they were still menstruating. If so, whether they were regular menstrual cycles, similarly to what had occurred during their reproductive life, They were classified as pre-menopausal women. If menstrual cycles were irregular in relation to flow and time intervals, they were classified as perimenopausal women. If they reported they did not menstruate, i.e., with amenorrhea for at least 12 consecutive months, they were classified as post-menopausal women [2]. 
To ensure the quality control of information, $10 \%$ of the interviews were redone through phone calls, using a simplified questionnaire. In this questionnaire, some perennial questions (with answer with no possibility of change since the time of the survey) were repeated by the research coordinators.

The typing of data followed the procedure of double entry, being performed with EPI-DATA 3.1 software. Comparisons of typing and analysis of consistency between them were made. Bivariate and multivariate analysis were performed in STATA 9.0 (Stata Corp., College Station, USA) software, according to a pre-established analysis plan.

The chi-square test and the test for linear trend were used to describe the characteristics of the sample according to menopausal status. The MetS components were also evaluated individually, both in terms of relative frequency and measures of central tendency and dispersion, according to menopausal status.

Estimates for crude and adjusted prevalence ratios with $95 \%$ confidence intervals were calculated by modified Poisson regression, with robust variance. Potential confounding factors in multivariable models were based on a conceptual framework from distal to proximal levels of MetS determination [24]. Variables were included and/or retained in models as potential confounding factors if they presented a p-value $<0.20$. Multivariate Model I was adjusted for the demographic variables (most distal level of determination); Model II was adjusted for the socioeconomic variables; Model III was adjusted for demographic and socioeconomic variables (only variables with $\mathrm{p}<0.20$ in Models I and II); and Model IV was adjusted for the most proximal determination variables (behavioral and reproductive variables) plus those variables socio-demographic variables kept as potential confounders in the multivariable analysis Model III $(p<0.20)$. Since nutritional status assessed by body mass index is directly related to MetS components, it was not included in any multivariate model. In each model, those variables with p-value $<0.05$ were considered statistically associated with the outcome.

This study was conducted according to the guidelines laid down in the Declaration of Helsinki and all procedures involving human subjects were approved by Research Ethics Committee of the University of Caxias do Sul, under opinion No. 124/08. Written informed consent was obtained from all subjects.

\section{Results}

During the collection period, from January 2010 to April 2011, 658 women who consulted in the clinic were eligible for the study; $41(6.2 \%)$ were refused and $66(10 \%)$ did not perform the laboratory tests. The following results refer to 551 women with average age of 51.1 years (sd 6.5).

Table 1 presents the sample characteristics and the distribution according to menopausal status. Most women said they were white and being married/in union, $28 \%$ had low education (less than 4 years of study) and $48.7 \%$ had low family income (less than 3 minimum wages). Sedentariness was present in $67.9 \%$ of the sample, and most women never smoked (52.6\%). As for reproductive variables, menarche between 12 and 13 years $(44.6 \%)$ and three or more pregnancies $(63.5 \%)$ prevailed in the sample. Most women (80.6\%) were overweight and obese.

Among all women in the study, $17 \%$ were pre-menopausal, $42 \%$ were perimenopausal and $38 \%$ were postmenopausal women. Table 1 shows, as expected, the advance of isolatedlymenopausal status as the age increases, and among women from 56 to 65 years, $88 \%$ were post-menopausal. Women with low education and without paid work were proportionally more in the postmenopausal period than the other groups of schooling and among those with paid work (Table 1).

The prevalence of MetS in the sample was $56.1 \%$ $\left(\mathrm{CI}_{95 \%}\right.$ : 51.9 to 60.2$)$, being more common among older women (56 to 65 years), with low education, menarche $\leq 11$ years old, with three or more pregnancies and in the post-menopausal period (Table 2). The prevalence of MetS increased gradually as menopausal status, showing a linear association, with $26 \%$ greater likelihood of MetS in post-menopausal women compared to pre-menopausal women (Table 2). Obese women had six times more MetS than the eutrophic women ( $78.8 \%$ vs. $13.1 \%)$.

Table 3 presents the multivariate models for MetS according to the three periods of the menopausal status. There was an increase of prevalence ratios when comparing perimenopause and post-menopause with premenopause; however, the confidence intervals include the unit (Table 3).

Figure 1 shows the isolated components of MetS according to the menopausal status. Among all women, the most prevalent altered components were: hypertension (84.8\%; $\mathrm{CI}_{95 \%}: 81.7$ to 87.8 ), waist circumference $(66.4 \%$; $\mathrm{CI}_{95 \%}$ : 62.5 to 70.4$)$ and HDL cholesterol $\left(51.7 \%\right.$; $_{95 \%}$ : 47.5 to 55.9$)$. The increase in the proportions of high blood pressure and high glucose showed a clear linear trend. The largest increase in waist circumference $(>88$ $\mathrm{cm}$ ) occurs between the pre-menopause to perimenopause, suggesting a later stabilization at a level slightly less than $70 \%$. The proportion of women with low HDL remained unchanged between the three periods.

Comparing the average values of the components according to the presence or absence of MetS (Table 4), it was observed that the largest increase was in values of serum triglycerides $(51.5 \%)$, followed by blood glucose $(21 \%)$. 
Table 1. Demographic, socioeconomic, behavior and reproductive characteristics of the sample and relationship with the menopausal status of women in climacteric period at a clinic in southern Brazil.

\begin{tabular}{|c|c|c|c|c|c|c|c|c|c|}
\hline $\begin{array}{l}\text { Variables } \\
\text { Age (years) quartile }\end{array}$ & \multicolumn{2}{|c|}{$\begin{array}{l}\text { Total n (\%) } \\
51(100)\end{array}$} & \multicolumn{2}{|c|}{$\begin{array}{c}\text { Pre n (\%) } \\
96(17.6)\end{array}$} & \multicolumn{2}{|c|}{$\begin{array}{l}\text { Peri n (\%) } \\
250(45.7)\end{array}$} & \multicolumn{2}{|c|}{$\begin{array}{l}\text { Post n (\%) } \\
201(36.7)\end{array}$} & $\begin{array}{l}\text { p-value } \\
<0.001^{* *}\end{array}$ \\
\hline 40 to 45 & 119 & $(21.6)$ & 47 & $(39.8)$ & 69 & $(58.5)$ & 2 & (1.7) & \\
\hline 46 to 50 & 155 & $(28.1)$ & 40 & $(25.8)$ & 100 & $(64.5)$ & 15 & $(9.7)$ & \\
\hline 51 to 55 & 143 & $(26.0)$ & 9 & $(6.4)$ & 65 & $(46.1)$ & 67 & $(47.5)$ & \\
\hline 56 to 65 & 134 & $(24.3)$ & 0 & $(0)$ & 16 & $(12.0)$ & 117 & $(88.0)$ & \\
\hline Skin color & & & & & & & & & $0.42^{*}$ \\
\hline White & 395 & $(71.8)$ & 74 & (18.9) & 176 & $(45.0)$ & 141 & $(36.1)$ & \\
\hline Not white & 155 & $(28.2)$ & 22 & $(14.2)$ & 74 & $(47.7)$ & 59 & $(38.1)$ & \\
\hline Marital status & & & & & & & & & $0.06^{*}$ \\
\hline Not married & 191 & $(34.7)$ & 25 & $(13.2)$ & 85 & $(44.7)$ & 80 & $(42.1)$ & \\
\hline Married/In union & 360 & $(65.3)$ & 71 & $(19.9)$ & 165 & $(46.2)$ & 121 & $(33.9)$ & \\
\hline Schooling (years) quartile & & & & & & & & & $0.03^{* *}$ \\
\hline 0 to 4 & 154 & $(28.1)$ & 16 & $(10.5)$ & 66 & $(43.1)$ & 71 & $(46.4)$ & \\
\hline 5 to 8 & 250 & $(45.5)$ & 53 & $(21.4)$ & 119 & $(48.0)$ & 73 & $(30.6)$ & \\
\hline 9 to 11 & 127 & $(23.1)$ & 24 & (19.9) & 53 & $(42.1)$ & 49 & $(38.9)$ & \\
\hline$\geq 12$ & 18 & (3.3) & 3 & $(16.7)$ & 11 & $(61.1)$ & 4 & $(22.2)$ & \\
\hline Family income (MS) quartile & & & & & & & & & $0.07^{* *}$ \\
\hline 0 to 2 & 153 & $(27.9)$ & 20 & $(13.1)$ & 70 & $(45.8)$ & 63 & $(41.2)$ & \\
\hline 2.01 to 3.00 & 114 & $(20.8)$ & 18 & $(15.9)$ & 52 & $(46.0)$ & 43 & $(38.1)$ & \\
\hline 3.01 to 5.00 & 151 & $(27.5)$ & 34 & (22.7) & 66 & $(44.0)$ & 50 & $(33.3)$ & \\
\hline 5.01 or more & 131 & $(23.9)$ & 23 & $(17.8)$ & 62 & $(48.1)$ & 44 & $(34.1)$ & \\
\hline Paid work & & & & & & & & & $<0.001^{*}$ \\
\hline No & 257 & $(46.6)$ & 41 & $(16.0)$ & 93 & $(36.3)$ & 122 & $(47.7)$ & \\
\hline Yes & 294 & $(53.4)$ & 55 & (18.9) & 157 & $(54.0)$ & 79 & $(27.1)$ & \\
\hline Physical activity & & & & & & & & & $0.002^{*}$ \\
\hline Sedentary & 374 & $(67.9)$ & 69 & $(18.6)$ & 184 & $(49.6)$ & 118 & $(31.8)$ & \\
\hline Non-sedentary & 177 & $(32.1)$ & 27 & $(15.3)$ & 66 & $(37.5)$ & 83 & $(47.2)$ & \\
\hline Smoking habit & & & & & & & & & $0.60^{*}$ \\
\hline Non-smoker & 290 & $(52.6)$ & 55 & $(19.0)$ & 125 & $(43.3)$ & 109 & $(37.7)$ & \\
\hline Former smoker & 158 & $(28.7)$ & 22 & $(14.0)$ & 77 & $(49.0)$ & 58 & $(36.9)$ & \\
\hline Smoker & 103 & $(18.7)$ & 19 & $(18.8)$ & 48 & $(47.5)$ & 34 & $(33.7)$ & \\
\hline Menarche (years) & & & & & & & & & $0.70^{*}$ \\
\hline$\geq 14$ & 190 & $(34.5)$ & 37 & $(19.5)$ & 82 & $(43.2)$ & 71 & $(37.4)$ & \\
\hline 12 to 13 & 246 & $(44.6)$ & 43 & $(17.8)$ & 110 & $(45.5)$ & 89 & $(36.8)$ & \\
\hline$\leq 11$ & 115 & (20.9) & 16 & (13.9) & 58 & $(50.4)$ & 41 & $(35.7)$ & \\
\hline
\end{tabular}


Continued

\begin{tabular}{|c|c|c|c|c|c|c|c|c|c|}
\hline Number of pregnancies & & & & & & & & & $<0.001^{*}$ \\
\hline 0 to 1 & 72 & $(13.1)$ & 23 & (31.9) & 20 & $(27.8)$ & 29 & $(40.3)$ & \\
\hline 2 & 129 & $(23.4)$ & 27 & $(21.1)$ & 53 & $(41.4)$ & 48 & $(37.5)$ & \\
\hline$\geq 3$ & 350 & $(63.5)$ & 46 & $(13.3)$ & 177 & $(51.0)$ & 124 & $(35.7)$ & \\
\hline Nutritional status (BMI) & & & & & & & & & $0.04^{* *}$ \\
\hline Eutrophic $(<24.9)$ & 107 & $(19.4)$ & 24 & $(22.6)$ & 43 & $(40.6)$ & 39 & $(36.8)$ & \\
\hline Overweight (25 to 29.9 ) & 189 & $(34.3)$ & 39 & $(20.7)$ & 89 & $(47.3)$ & 60 & $(31.9)$ & \\
\hline Obesity $(\geq 30)$ & 255 & $(46.3)$ & 33 & $(13.0)$ & 118 & $(46.6)$ & 102 & $(40.3)$ & \\
\hline
\end{tabular}

" p-value for Pearson, ${ }^{* *}$ p-value Linear Association.

Table 2. Prevalence and prevalence ratios of Metabolic Syndrome according to demographic, socioeconomic, behavioral, reproductive and nutritional status characteristics of women in climacteric period at a clinic in southern Brazil (n= 551 women).

\begin{tabular}{|c|c|c|c|c|c|c|}
\hline Variables & \multicolumn{2}{|c|}{ MetS n (\%) } & \multirow{2}{*}{$\frac{\text { p-value }}{0.04^{* *}}$} & \multirow{2}{*}{ PR } & \multirow[t]{2}{*}{ CI (95\%) } & \multirow{2}{*}{$\begin{array}{c}\text { p-value } \\
0.04\end{array}$} \\
\hline & & & & & & \\
\hline 40 to 45 & 60 & $(50.4)$ & & 1 & & \\
\hline 46 to 50 & 85 & $(54.8)$ & & 1.09 & $0.87-1.35$ & \\
\hline 51 to 55 & 78 & $(54.5)$ & & 1.08 & $0.86-1.37$ & \\
\hline 56 to 65 & 86 & $(64.2)$ & & 1.27 & $1.02-1.58$ & \\
\hline Skin color & & & $0.47^{*}$ & & & 0.50 \\
\hline White & 225 & $(57.0)$ & & 1 & & \\
\hline Not white & 83 & $(53.5)$ & & 0.94 & $0.79-1.11$ & \\
\hline Marital status & & & $0.10^{* *}$ & & & 0.11 \\
\hline Not married & 98 & $(51.3)$ & & 1 & & \\
\hline Married/in union & 211 & $(58.6)$ & & 1.14 & $0.97-1.34$ & \\
\hline Schooling (years) quartile & & & $0.04^{* *}$ & & & 0.04 \\
\hline 0 to 4 & 97 & $(63.0)$ & & 1 & & \\
\hline 5 to 8 & 137 & $(54.8)$ & & 0.87 & $0.74-1.03$ & \\
\hline$\geq 9$ & 74 & $(51.0)$ & & 0.81 & $0.66-0.99$ & \\
\hline Family Income (MS R\$510) quartile & & & $0.04^{*}$ & & & 0.06 \\
\hline 0 to 2 & 87 & $(56.9)$ & & 1 & & \\
\hline 2.01 to 3.00 & 75 & $(65.8)$ & & 1.16 & $0.96-1.40$ & \\
\hline 3.01 to 5.00 & 83 & $(55.0)$ & & 0.97 & $0.79-1.18$ & \\
\hline 5.01 or more & 62 & $(47.3)$ & & 0.83 & $0.66-1.04$ & \\
\hline Paid work & & & $0.18^{*}$ & & & 0.18 \\
\hline No & 152 & $(59.1)$ & & 1 & & \\
\hline Yes & 157 & $(53.4)$ & & 0.90 & $0.78-1.05$ & \\
\hline Physical activity & & & $0.38^{*}$ & & & 0.38 \\
\hline Sedentary & 205 & $(54.8)$ & & 1 & & \\
\hline Non-sedentary & 104 & $(58.8)$ & & 1.07 & $0.92-1.25$ & \\
\hline Smoking habit & & & $0.02^{*}$ & & & 0.10 \\
\hline
\end{tabular}




\section{Continued}

\begin{tabular}{|c|c|c|c|c|c|c|}
\hline Non-smoker & 166 & $(57.2)$ & & 1 & & \\
\hline Former smoker & 97 & $(61.4)$ & & 1.07 & $0.91-1.26$ & \\
\hline Smoker & 46 & $(44.7)$ & & 0.78 & $0.62-0.99$ & \\
\hline Menarche (years) & & & $0.05^{* *}$ & & & 0.04 \\
\hline$\geq 14$ & 100 & $(52.6)$ & & 1 & & \\
\hline 12 to 13 & 134 & $(54.5)$ & & 1.03 & $0.87-1.24$ & \\
\hline$\leq 11$ & 75 & $(65.2)$ & & 1.24 & $1.02-1.50$ & \\
\hline Number of pregnancies & & & $0.01^{* *}$ & & & 0.02 \\
\hline 0 to 1 & 34 & $(47.2)$ & & 1 & & \\
\hline 2 & 64 & $(49.6)$ & & 1.05 & $0.78-1.42$ & \\
\hline$\geq 3$ & 211 & $(60.3)$ & & 1.28 & $0.99-1.65$ & \\
\hline Nutritional status (BMI) & & & $<0.001^{* *}$ & & & $<0.001$ \\
\hline Eutrophic $(<24.9)$ & 14 & $(13.1)$ & & 1 & & \\
\hline Overweight (25 to 29.9 ) & 94 & $(49.7)$ & & 3.80 & $2.28-6.33$ & \\
\hline Obesity $(\geq 30)$ & 201 & $(78.8)$ & & 6.02 & $3.68-9.86$ & \\
\hline Menopausal status & & & $0.05^{* *}$ & & & 0.06 \\
\hline Pre-menopause & 46 & $(47.9)$ & & 1 & & \\
\hline Perimenopause & 140 & $(56.0)$ & & 1.17 & $0.92-1.48$ & \\
\hline Post-menopause & 121 & $(60.2)$ & & 1.26 & $0.99-1.60$ & \\
\hline
\end{tabular}

p-value for Pearson, ${ }^{* *}$ p-value Linear Association.

Table 3. Multivariate models and $\mathrm{CI}_{95 \%}$ of MetS in relation to menopausal status in women in climacteric period at a clinic in southern Brazil.

\begin{tabular}{ccccc}
\hline Exposures & Model I & Model II & Model III & Model IV \\
\hline p-value & 0.38 & 0.12 & 0.47 & 0.55 \\
Pre & 1 & 1 & 1 & 1 \\
Peri & $1.15(0.90-1.46)$ & $1.16(0.92-1.48)$ & $1.13(0.87-1.44)$ & $1.08(0.85-1.38)$ \\
Post & $1.15(0.85-1.55)$ & $1.22(0.96-1.57)$ & $1.12(0.83-1.52)$ & $1.10(0.81-1.48)$ \\
\hline
\end{tabular}

I — Controlled for age and marital status, II — Controlled for schooling, income, and paid work, III-Controlled for age, marital status and schooling, IV - Controlled for age, marital status, schooling smoking habit, menarche and number of pregnancies.

Table 4. Mean values and standard deviation (sd) of the components of MetS in women treated at a climacteric clinic in southern Brazil according to the presence or absence of MetS.

\begin{tabular}{lcccc}
\hline \multicolumn{1}{c}{ Component } & $\begin{array}{c}\text { Without MetS (n=242) } \\
\text { mean (sd) (a) }\end{array}$ & $\begin{array}{c}\text { With MetS } \\
(307) \text { mean (sd) (b) }\end{array}$ & Variation (a:b) & P-value \\
\hline Diastolic BP & $137.2( \pm 17.87)$ & $145.6( \pm 18.98)$ & $\uparrow 6.1 \%$ & $<0.001$ \\
Systolic BP & $87.2( \pm 12.21)$ & $92.2( \pm 12.67)$ & $\uparrow 5.7 \%$ & $<0.001$ \\
Waist circumference & $86.7( \pm 11.70)$ & $101.0( \pm 12.20)$ & $\uparrow 16.5 \%$ & $<0.001$ \\
HDL & $57.1( \pm 11.97)$ & $46.4( \pm 10.75)$ & $\downarrow 18.7 \%$ & $<0.001$ \\
Triglycerides & $111.5( \pm 52.41)$ & $168.9( \pm 74.54)$ & $\uparrow 51.5 \%$ & $<0.001$ \\
Glucose & $88.5( \pm 10.37)$ & $107.0( \pm 36.40)$ & $\uparrow 20.9 \%$ & $<0.001$ \\
\hline
\end{tabular}

Test: t-test, BP: blood pressure, HDL: high density lipoprotein. 


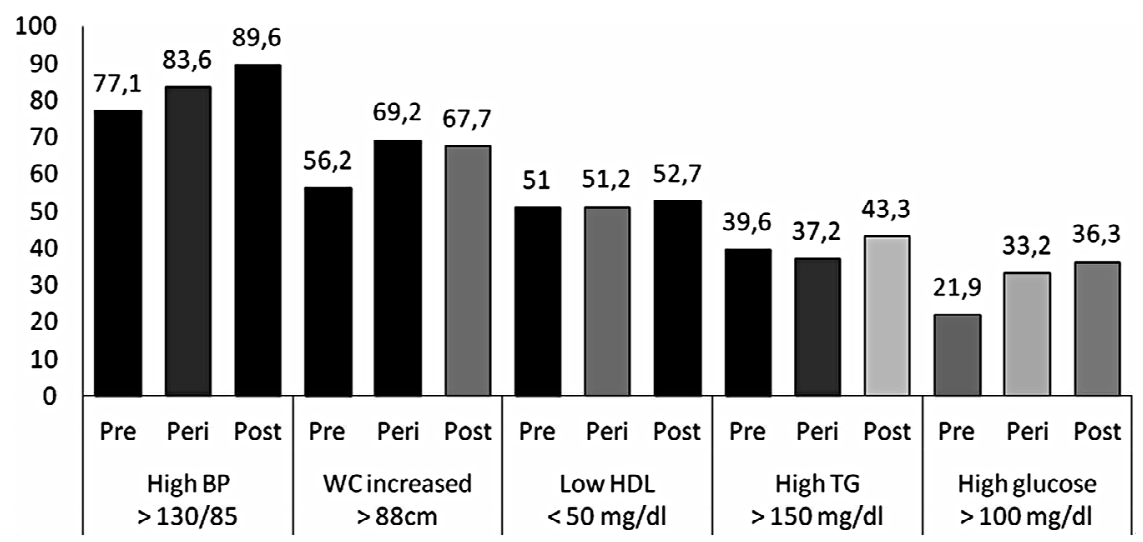

HDL (high density lipoprotein) and TG (triglycerides): p-value $>0.05$, BP (blood pressure) and glucose: p-value $<0.05$ (linear trend), WC (waist circumference): $\mathrm{p}$-value $=0.07$ (Pearson).

Figure 1. Prevalence of altered components of MetS according to menopausal status in women in climacteric period at a clinic in southern Brazil $(n=551)$.

\section{Discussion}

The present study investigated the relationship between menopausal status and presence of Metabolic Syndrome in women from 40 to 65 years treated at a clinic of climacteric and Gynecological Surgery in southern Brazil. The prevalence of MetS in this population was high, showing an increasing trend in relation to periods of pre-, peri- and post-menopause. In the multivariate model, this trend lost statistical significance. Among MetS components isolatedly, there was a linear increase in the occurrence of high blood pressure and high glucose over the three periods of the menopausal status, but the proportion of low HDL remained unchanged. Comparing means of each component between women with and without MetS, the biggest variation was in the mean value triglycerides ( $51 \%$ increased), followed by glucose $(21 \%)$.

The study found a prevalence of MetS of $56.1 \%$, considerably higher than other studies conducted in clinics and similar to population studies in Brazil. Cross-sectional population-based studies showed prevalence similar to that found in our study-56.9\% (among women over 45 years) in the semi-arid region of Bahia [25] and $59.9 \%$ (in women over 60 years) in Novo Hamburgo, state of Rio Grande do Sul [26]. However, Figueiredo Neto [8], investigating women in climacteric period in a gynecology clinic in Maranhão, found lower prevalence, of $34.7 \%$ [8]. This study, like ours, was performed in public health service, with the average age of women of 49.7 years, slightly lower than ours.

Just as within the country, the prevalence of MetS in women in climacteric period varies widely around the world. In Taiwan [17] and Korea [12], in studies performed in clinics, the prevalence of MetS were even lower ( $6.2 \%$ and $17.9 \%$, respectively). On the other hand, population-based studies show higher prevalence rates of MetS. In Iran, Ainy et al. [18] conducted a study with
2182 women in menopausal transition and found a prevalence of MetS of $63 \%$. In addition to differences in culture and lifestyle, which are not evaluated, the difference between the prevalence on this study and ours may be partly attributed to the higher age averages of the study performed in Iran.

In our study, an increase in the prevalence of Metabolic Syndrome was observed between the periods of pre- to peri- and post-menopause, although this effect has missed statistical significance in multivariate analysis. These findings are consistent with recent studies that demonstrate increased prevalence of MetS in women in climacteric period, and a greater increase in post-menopause when compared to pre-menopause was observed [8,11,12,20,27]. Miller et al. [28], through a crosssectional population-based study in the USA women with a cohort of immigrant women from the Soviet Union, presented an even bigger difference than the other studies in the prevalence of MetS in the period (13\% in premenopause, $68 \%$ in post-menopause). This increased prevalence in post-menopause may have occurred because the average age was higher in the study by Miller (57.3 years) when compared with the average age of our study (51.1 years). In a study performed on a gynecology clinic in Brazil [8], with women with an average age of 49.7 years, the prevalence of MetS in pre-menopausal women was $24 \%$, while in post-menopausal women was $44.4 \%$, prevalence lower than of our study. These findings show the age as a participating factor when the relationship of menopausal status and the occurrence of MetS is studied. Although the risk of MetS increases with age, cross-sectional population-based and cohort studies show that post-menopausal women are at greater risk of having MetS, regardless of age [9,11,29].

Establishing the effect of climacteric as an independent risk factor for MetS has been a tough challenge to 
researchers, given the difficulty of separating the effects of natural aging process of menopause [27]. The predominance of testosterone is being indicated as a hormonal change that is directly associated with the incidence of MetS, regardless of aging and other standard risk factors of CVD [29]. Other studies support the hypothesis that the reduction of estrogen would be the main factor for the increase of this incidence, directly associated with aging and biological aging, which itself already contributes to the increase of health problems. However, Kok et al. (2006) [30] conducted a study with Framingham's data to investigate whether cardiovascular risk profile could accelerate menopause. The results showed that the risk of heart disease determines the age of menopause. This offers an explanation for the inconsistent results in the cardiovascular disease rates in relation to the age of menopause.

By investigating the characteristics of women, it was observed that there was a similar behavior in association with both the MetS outcome and the menopausal status exposure, namely: age, schooling, number of pregnancies and nutritional status. Moreover, menarche was only associated with MetS, not with menopausal status.

Socioeconomic characteristics such as low schooling and income are factors associated with an increased prevalence of MetS in most studies that evaluate women in climacteric period [29,31]. Romaguera et al. [31], using data from a longitudinal study, showed that higher schooling seems to provide a protective effect, even after adjustment for confounding factors. Number of pregnancies has often been studied in relation to MetS. By assessing Iranian women, Mousavi et al. [32] found that the number of pregnancies in women with MetS was significantly higher than in those without MetS (5.2 \pm 3.1 vs. $3.5 \pm 2.6, \mathrm{p}<0.001)$. The prevalence of MetS in nulliparous women, mothers with 1 - 4 children and more than 4 children was $15.2 \%, 26.2 \%$ and $49.6 \%$, respectively. As to menarche, Feng et al. [19] also found that women with early menarche ( 8 - 13 years) have significantly increased risk for MetS $\left(\mathrm{OR}=1.32 ; \mathrm{CI}_{95 \%}: 1.14-1.53\right)$ than women with menarche from 14 years. Heys et al. [33] evaluated the effect of menarche in the development of MetS in 7349 Chinese women with 50 years or more of a historical cohort. Adjusted for age, schooling and number of pregnancies, the lower age of menarche $(<12.5$ years $)$, when compared to the older $(\geq 14.5$ years $)$, was associated with a higher risk of MetS $(\mathrm{OR}=1.49$; $\mathrm{CI}_{95 \%}$ : 1.22 - 1.82). Regarding the nutritional status, obese and overweight women had higher prevalence rates of MetS when compared with eutrophic women. One of the components of MetS is abdominal obesity, directly related to overall obesity. In a study conducted with the 1982 Pelotas cohort, obesity increased the risk of young adults having MetS by about 40 times [34].
As for the prevalence of MetS components, altered blood pressure was the most common characteristic observed in the population studied, followed by increased waist circumference and low HDL. Other studies showed that waist increased circumference and blood pressure had the highest prevalence among the MetS components, suggesting that these are the components that most influence the prevalence of MetS $[9,12]$. Specifically in relation to blood pressure, the region where the study was conducted is considered one of the biggest consumers of sodium-rich foods and, consequently, a high prevalence of hypertension is found in this population. While the average sodium consumption in Brazil is $4.5 \mathrm{~g}$, in southern Brazil is $5 \mathrm{~g}$ [35]. A national population survey showed that the female population that resides in southern Brazil consumes 64\% more sodium than the recommended [36].

When the occurrence of the altered components during different periods of climacteric was observed, there were gradual increases in the prevalence of altered blood pressure and high glucose when comparing pre-, periand post-menopause periods. The increase in blood pressure during climacteric is common in many studies in different countries $[8,9,12,17]$. Sièminksa et al. [16], investigating Polish women, found in pre-menopausal women a prevalence of $7 \%$ of hypertension, while in post-menopausal women this percentage increased to $30 \%$. High glucose (above $100 \mathrm{mg} / \mathrm{dl}$ ) has also increased its prevalence in climacteric period $[8,9,11]$. In a study by Kim et al. [9], with Korean women, the prevalence found in pre-menopausal women was $9.8 \%$, while among post-menopausal women the prevalence of altered glucose was $26 \%$. It was observed that high waist circumference had its highest prevalence during the perimenopause period. Few studies assess the perimenopause; most evaluates the difference between pre- and postmenopause. Low HDL cholesterol did not present change in prevalence between the three periods.

In our study, the average values of the components were evaluated according to the presence or absence of MetS. An increase in the average value of those with MetS was observed. The biggest variation (increase of $51 \%$ ) occurred in the average of serum triglycerides, 111.5 vs. 158.9 , respectively, in women without and with MetS-followed by blood glucose (increase of $20 \%$ ) and serum HDL cholesterol (reduction of 18\%). The smallest average variation occurred in the blood pressure levels, an increase of $6 \%$ among women without and with MetS. In the study by Janssen [29], which also compared the data between women with and without MetS, the same trend in the variation of components is observed. It is noteworthy that in our study the averages in women with and without MetS are higher, and all the component values among women with MetS were higher than the rec- 
ommended by NCEP [3]. These results reinforce the need to investigate the components of MetS both aggregate and isolatedly in this period of life of women.

Our findings should be interpreted with some observations. First, the way in which the three periods of menopausal status were analyzed, i.e., based on self-reported information. Perimenopausal women reported being with irregular menstrual cycles or less than twelve months without menstruating. Since this is a cross-sectional study, we only have information from the current moment of these women. One year after the collection, we contacted 55 women, who at the time of the survey reported that were not menstruating for less than 12 months, which were classified in perimenopause for that reason. Even classifying as post-menopausal the women who reported being more than 12 months without menstruating at the time of phone call, the analysis showed no differences. Therefore, we chose to use the data collected during the interview. A second aspect that deserves attention refers to the sample size both in terms of total size and the number within the categories of exposure. In subsequent calculations we found that 594 women would be needed to identify significant difference of MetS between pre- and post-menopause. In addition, we obtained a small number of classified women in the pre-menopause period, and in our sample calculation, we expected to find a greater number of these women. Most of our sample was in perimenopause. It is also important to assess that, among those who were post-menopausal, the average age was 57 years $( \pm 4.9)$, demonstrating that exposure to post-menopause on these women was small. In several studies the average age in post-menopause is greater than what was found in our study $[12,18,20]$. The study by Cho et al. [12] presents results that support the hypothesis that the risk of having MetS increases with the years since menopause, and tends to decrease after 14 years from the last menstruation. The highest prevalence of MetS would be around 60 years, value greater than the average age we found. By analyzing the results of these other studies, it is possible to infer that there were no statistically significant differences in our multivariate analysis because our sample has a greater number of perimenopausal women and because of the small exposure of women to menopause.

Another point to highlight relates to the generalization of our findings, mainly regarding the prevalence of MetS. Our sample came from a health service specialized in women in climacteric period, which serve only users of the Unified Health System, with low schooling and income. Since this sample is not population-based, the results should not be generalized to the general population.

The last observation is about the percentage of rejections and losses caused by failure to carry out the laboratory tests. However, in a comparative analysis of the main characteristics of the studied group (551) and such losses/rejections, there were no statistically significant differences between the characteristics investigated.

\section{Conclusions}

This study stands out not only for being one of the few studies conducted in Brazil in a secondary level public clinic, as well as for evaluating the relationship of menopausal status with each of the isolated components of MetS. Thus, it was possible to demonstrate that, in addition to blood pressure and waist circumference, the change in blood glucose and serum triglycerides play an important role in increasing Metabolic Syndrome during climacteric.

The current study indicates variation on the distribution of MetS and each components according to menopausal status and other women characteristics. The combination of these findings suggests that future studies on MetS should also have foresight to investigate the relationship of MetS components isolated according to the women characteristics. Certainly, this type of approach should improve understanding and targeting of actions and programs focusing on women in this period of life.

\section{Acknowledgments}

Clinic of Climacteric and of Gynecological Surgery-Central Clinic of the University of Caxias do Sul; National Council of Technological and Scientific Development (CNPq PQ n.304793/2010-8).

\section{REFERENCES}

[1] WHO, "Research on the Menopause," Geneva, 1981.

[2] WHO, "Research on the menopause in the 1990," Geneva, 1996.

[3] "Executive Summary of the Third Report of the National Cholesterol Education Program, NCEP Expert Panel on Detection, Evaluation, and Treatment of High Blood Cholesterol in Adults (Adult Treatment Panel III)," JAMA, Vol. 285, No. 19, 2001, pp. 2486-2497. doi:10.1001/jama.285.19.2486

[4] E. S. Ford, W. H. Giles and W. H. Dietz, "Prevalence of the Metabolic Syndrome among US Adults: Findings from the Third National Health and Nutrition Examination Survey," JAMA, Vol. 287, No. 3, 2002, pp. 356-359. doi:10.1001/jama.287.3.356

[5] J. B. Meigs, P. W. Wilson, D. M. Nathan, R. B. Sr. D'Agostino, K. Williams and S. M. Haffner, "Prevalence and Characteristics of the Metabolic Syndrome in the San Antonio Heart and Framingham Offspring Studies," Diabetes, Vol. 52, No. 8, 2003, pp. 2160-2167. doi:10.2337/diabetes.52.8.2160

[6] M. A. Nakazone, A. Pinheiro, M. C. Braile, M. A. Pinhel, G. F. de Sousa, S. Pinheiro Jr., et al., "Prevalence of Metabolic Syndrome Using NCEP-ATPIII and IDF Defini- 
tions in Brazilian Individuals," Revista Da Associacao Medica Brasileira, Vol. 53, No. 5, 2007, pp. 407-413. doi:10.1590/S0104-42302007000500016c

[7] L. B. Salaroli, G. C. Barbosa, J. G. Mill and M. C. B. Molina, "Prevalence of Metabolic Syndrome in PopulationBased Study, Vitoria, ES-Brazil," Brazilian Archives of Endocrinology \& Metabolism, Vol. 51, No. 7, 2007, pp. 1143-1152. doi:10.1590/S0004-27302007000700018

[8] J. A. F. Neto, E. D. Figueredo, J. B. Barbosa, F. B. Fde, G. R. Costa, V. J. Nina, et al., "Metabolic Syndrome and Menopause: Cross-Sectional Study in Gynecology Clinic," Brazilian Archives of Cardiology, Vol. 95, No. 3, 2010, pp. 339-345. doi:10.1590/S0066-782X2010005000094

[9] H. M. Kim, J. Park, S. Y. Ryu and J. Kim, "The Effect of Menopause on the Metabolic Syndrome among Korean Women: The Korean National Health and Nutrition Examination Survey, 2001," Diabetes Care, Vol. 30, No. 3, 2007, pp. 701-706. doi:10.2337/dc06-1400

[10] I. Janssen, L. H. Powell, S. Crawford, B. Lasley and K. Sutton-Tyrrell, "Menopause and the Metabolic Syndrome: The Study of Women's Health across the Nation," Archives of Internal Medicine, Vol. 168, No. 14, 2008, pp. 15681575. doi:10.1001/archinte.168.14.1568

[11] R. Eshtiaghi, A. Esteghamati and M. Nakhjavani, "Menopause is an Independent Predictor of Metabolic Syndrome in Iranian women," Maturitas, Vol. 65, No. 3, 2010, pp. 262-266. doi:10.1016/j.maturitas.2009.11.004

[12] G. J. Cho, H. T. Park, J. H. Shin, T. Kim, J. Y. Hur, Y. T. Kim, et al., "The Relationship between Reproductive Factors and Metabolic Syndrome in Korean Post-Menopausal Women: Korea National Health and Nutrition Survey 2005," Menopause, Vol. 16, No. 5, 2009, pp. 9981003. doi:10.1097/gme.0b013e3181559860

[13] F. Azizi and E. Ainy, "Coronary Heart Disease Risk Factors and Menopause: A Study in 1980 Tehranian Women, the Tehran Lipid and Glucose Study," Climacteric, Vol. 6, No. 4, 2003, pp. 330-336. doi:10.1080/cmt.6.4.330.336

[14] K. A. Matthews, S. L. Crawford, C. U. Chae, S. A. Everson-Rose, M. F. Sowers, B. Sternfeld, et al., "Are Changes in Cardiovascular Disease Risk Factors in Midlife Women Due to Chronological Aging or to the Menopausal Transition?" Journal of the American College of Cardiology, Vol. 54, No. 25, 2009, pp. 2366-2373. doi:10.1016/j.jacc.2009.10.009

[15] A. Ghosh, "Comparison of Risk Variables Associated with the Metabolic Syndrome in Pre- and Post-Menopausal Bengalee Women," Cardiovascular Journal of Africa, Vol. 19, No. 4, 2008, pp. 183-187.

[16] L. Sieminska, C. Wojciechowska, W. Foltyn, D. Kajdaniuk, B. Kos-Kudla, B. Marek, et al., "The Relation of Serum Adiponectin and Leptin Levels to Metabolic Syndrome in Women before and after the Menopause," Polish Journal of Endocrinology, Vol. 57, No. 1, 2006, pp. 1522 .

[17] W. Y. Lin, W. S. Yang, L. T. Lee, C. Y. Chen, C. S. Liu, C. C. Lin, et al., "Insulin Resistance, Obesity, and Metabolic Syndrome among Non-Diabetic Pre- and PostMenopausal Women in North Taiwan," International Journal of Obesity, Vol. 30, No. 6, 2006, pp. 912-917. doi:10.1038/sj.ijo.0803240

[18] E. Ainy, P. Mirmiran, S. Zahedi Asl and F. Azizi, "Prevalence of Metabolic Syndrome during Menopausal Transition Tehranian Women: Tehran Lipid and Glucose Study (TLGS)," Maturitas, Vol. 58, No. 2, 2007, pp. 150-155. doi:10.1016/j.maturitas.2007.07.002

[19] Y. Feng, X. Hong, E. Wilker, Z. Li, W. Zhang, D. Jin, et al., "Effects of Age at Menarche, Reproductive Years, and Menopause on Metabolic Risk Factors for Cardiovascular Diseases," Atherosclerosis, Vol. 196, No. 2, 2008, pp. 590597. doi:10.1016/j.atherosclerosis.2007.06.016

[20] P. Henneman, A. C. Janssens, M. C. Zillikens, M. Frolich, R. R. Frants, B. A. Oostra, et al., "Menopause Impacts the Relation of Plasma Adiponectin Levels with the Metabolic Syndrome," Journal of Internal Medicine, Vol. 267, No. 4, 2010, pp. 402-409. doi:10.1111/j.1365-2796.2009.02162.x

[21] P. Marques-Vidal, M. Bochud, V. Mooser, F. Paccaud, G. Waeber and P. Vollenweider, "Prevalence of Obesity and Abdominal Obesity in the Lausanne Population," BMC Public Health, Vol. 8, No. 330, 2008, p. 330. doi:10.1186/1471-2458-8-330

[22] WHO, "Obesity: Preventing, and Managing the Global Epidemic," Geneva, 1998.

[23] P. Palatini, F. Dorigatti, E. Bonso and F. Ragazzo, "Validation of Microlife BP W100 Wrist Device Assessed according to the European Society of Hypertension and the British Hypertension Society Protocols," Blood Pressure Monitoring, Vol. 14, No. 1, 2009, pp. 41-44. doi:10.1097/MBP.0b013e32831e30d2

[24] C. G. Victora, S. R. Huttly, S. C. Fuchs and M. T. Olinto, "The Role of Conceptual Frameworks in Epidemiological Analysis: A Hierarchical Approach," International Journal of Epidemiology, Vol. 26, No. 1, 1997, pp. 224-227. doi:10.1093/ije/26.1.224

[25] E. P. S. M. Oliveira and M. D. A. Lima, "Prevalence of Metabolic Syndrome in a Semi-arid Rural Area in Bahia," Brazilian Archives of Endocrinology \& Metabolism, Vol. 50, No. 3, 2006, pp. 456-465. doi:10.1590/S0004-27302006000300008

[26] J. L. Rigo, R. R. Lacorte, J. L. Vieira and C. L. Reichert, "Prevalence of Metabolic Syndrome in an Elderly Community: Comparison between Three Diagnostic Methods," Brazilian Archives of Cardiology, Vol. 93, No. 2, 2009, pp. 85-91. doi:10.1590/S0066-782X2009000800004

[27] V. R. Mesch, L. E. Boero, N. O. Siseles, M. Royer, M. Prada, F. Sayegh, et al., "Metabolic Syndrome throughout the Menopausal Transition: Influence of Age and Menopausal Status," Climacteric, Vol. 9, No. 1, 2006, pp. 4048. doi:10.1080/13697130500487331

[28] A. M. Miller, J. Wilbur, P. J. Chandler and O. Sorokin, "Cardiovascular Disease Risk Factors and Menopausal Status in Midlife Women from the Former Soviet Union," Women Health, Vol. 38, No. 3, 2003, pp. 19-36. doi:10.1300/J013v38n03 03

[29] I. Janssen, L. H. Powell, R. Kazlauskaite and S. A. Dugan, "Testosterone and Visceral Fat in Midlife Women: The Study of Women's Health across the Nation (SWAN) Fat Patterning Study," Obesity, Vol. 18, No. 3, 2010, pp. 604- 


\section{0. doi:10.1038/oby.2009.251}

[30] H. S. Kok, K. M. van Asselt, Y. T. van der Schouw, I. van der Tweel, P. H. Peeters, P. W. Wilson, et al., "Heart Disease Risk Determines Menopausal Age rather than the Reverse," Journal of the American College of Cardiology, Vol. 47, No. 10, 2006, pp. 1976-1983.

doi:10.1016/j.jacc.2005.12.066

[31] J. Romaguera, A. P. Ortiz, F. J. Roca, G. Colon and E. Suarez, "Factors Associated with Metabolic Syndrome in a Sample of Women in Puerto Rico," Menopause, Vol. 17, No. 2, 2010, pp. 388-392. doi:10.1097/gme.0b013e3181bd5393

[32] E. Mousavi, M. Gharipour, A. Tavassoli, G. H. Sadri and N. Sarrafzadegan, "Multiparity and Risk of Metabolic Syndrome: Isfahan Healthy Heart Program," Metabolic Syndrome and Related Disorders, Vol. 7, No. 6, 2007, pp. 519-524. doi:10.1089/met.2008.0076
[33] M. Heys, C. M. Schooling, C. Jiang, B. J. Cowling, X. Lao, W. Zhang, et al., "Age of Menarche and the Metabolic Syndrome in China," Epidemiology, Vol. 18, No. 6, 2007, pp. 740-746. doi:10.1097/EDE.0b013e3181567faf

[34] V. M. Silveira, B. L. Horta, D. P. Gigante and M. R. Azevedo Junior, "Metabolic Syndrome in the 1982 Pelotas Cohort: Effect of Contemporary Lifestyle and Socioeconomic Status," Brazilian Archives of Endocrinology \& Metabolism, Vol. 54, No. 4, 2010, pp. 390-397. doi:10.1590/S0004-27302010000400008

[35] F. Sarno and C. A. Monteiro, "Relative Importance of Body Mass Index and Waist Circumference for Hypertension in Adults," Journal of Public Health, Vol. 41, No. 5, 2007, pp. 788-796. doi:10.1590/S0034-89102007000500013

[36] IBGE, "Research Budgets Family 2008-2009: Food Consumption Analysis Staff in Brazil," Rio de Janeiro, 2011. 